\title{
Pediatric Ethics and Communication Excellence (PEACE) Rounds: Decreasing Moral Distress and Patient Length of Stay in the PICU
}

Lucia Wocial, Veda Ackerman, Brian Leland, Brian Benneyworth, Vinit Patel, Yan Tong, Mara Nitu

Abstract

Innovation: Establish formal weekly discussions of patients with prolonged PICU stay to reduce healthcare providers' moral distress and decreases length of stay for patients with lifethreatening illnesses.

Evaluation: Pre/post intervention design measuring provider moral distress and comparing patient outcomes using retrospective historical controls.

Setting: Pediatric Intensive Care Unit in a quaternary care Children's Hospital.

Participants: Physicians and nurses on staff in the unit.

Patients: There were 60 patients in the interventional and 66 patients in the historical control group.

Intervention: Over a year, weekly meetings (PEACE rounds) to establish goals of care for patients with longer than 10 days length of stay in the ICU.

Results: Moral distress scores measured intermittently with the MDT fluctuated. "Clinical situations" represented the most frequent contributing factor to moral distress. Post intervention, overall MDS-R scores were lower for respondents in all categories (non-significant), and on three specific items (significant). Patient outcomes before and after PEACE intervention showed a statistically significant decrease in PRISM indexed LOS (4.94 control vs 3.37 PEACE, $\mathrm{p}=0.015)$, a statistically significant increase in both code status changes DNR (11\% control, $28 \%$ PEACE, $\mathrm{p}=0.013)$, and in-hospital death (9\% control, 25\% PEACE, $\mathrm{p}=0.015)$, with no change in patient 30 or 365 day mortality.

Conclusion: The addition of a clinical ethicist and senior intensivist to weekly interprofessional team meetings facilitates difficult conversations regarding realistic goals of care. The PEACE

This is the author's manuscript of the article published in final edited form as: Wocial, L., Ackerman, V., Leland, B., Benneyworth, B., Patel, V., Tong, Y., \& Nitu, M. (2017). Pediatric Ethics and Communication Excellence (PEACE) Rounds: Decreasing Moral Distress and Patient Length of Stay in the PICU. HEC Forum, 29(1), 75-91. https://doi.org/10.1007/s10730-016-9313-0 
intervention had a positive impact on some factors that contribute to moral distress and can shorten PICU length of stay for some patients. 


\section{Pediatric Ethics And Communication Excellence (PEACE) Rounds: Decreasing Moral Distress and Patient Length of Stay in the PICU}

When children are at risk of death or severely impaired functional recovery even with aggressive life sustaining treatment, some parents may feel the harm or burden outweighs the benefit of continuing such treatment. In such circumstances, offering comfort care is ethically acceptable (American Academy of Pediatrics, 1996) and consistent with recommendations from the "Choosing Wisely" campaign (Halpern et al, 2014). However when it comes to the care of children, doing anything less than "everything" can be difficult (Clark and Dudzinski, 2013). In many cases, even when physicians believe the child will not survive his/her ICU experience, placing limits on interventions that offer marginal benefit, or merely prolong the suffering of a child is often not openly discussed either within the health care team or between the team and the patient's family. Avoidance of discussions about setting limits may lead to unnecessary additional medical interventions, a common occurrence associated with higher levels of moral distress felt by members of the health care team (Mekechuk, 2006, Trotochaud et al, 2013; Wilson et. al, 2013).

Moral distress (MoD) is a complex construct with considerable debate in the literature on an exact definition (Fourie, 2015: Musto et al, 2015). For this project, we defined MoD as a feeling that occurs when an individual's moral integrity is seriously compromised, either because one feels unable to act in accordance with core values and obligations, or attempted actions fail to achieve the desired outcome (Whitehead et al, 2015). Put more simply, it is the experience of believing one knows the ethically correct thing to do however something or someone prevents the individual from acting (Wocial and Weaver, 2013). The presence of moral distress should serve as an alarm signal indicating conscientious persons are practicing in challenging contexts (Garros et al, 2015) and failing to address this may have negative consequences (Halpern, 2011).

The concept of MoD originated in nursing however a growing body of research confirms that it is not only experienced by individuals across disciplines in healthcare (Allen et al, 2013: Bruce, 2015: Uhrich et al, 2010), it has negative consequences for healthcare providers and patients (Elpern et al, 2005: Houston et al, 2013: Whitehead et al, 2015). MoD is often associated with what providers perceive as futile or inappropriate treatment (Mobley et al, 2007: Piers et al, 2011; Wilson et al, 2013) and poor physician nurse collaboration (Hamric and 
Blackhall, 2007: Kalvemark et al, 2004: McAndrew et al 2011: Papathanassoglou et al, 2012). Because high levels are consistently associated with poor ethical climate of working environments, it may act as an indirect indicator of quality patient care (Lamiani, Borghi, \& Argentero, 2015; Wall et al, 2015). There is some indication that promoting open discussion about ethically challenging situations will lower moral distress (Bruce et al, 2014: Karanikola et al, 2014; Wocial et al, 2010).

Processes and supports are needed to foster open discussion when healthcare providers have ethical concerns (Garros et al, 2015). A clinical ethicist can promote genuine dialogue between health care professionals who must explore difficult choices when caring for patients who experience life-threatening illness (Bruce et al, 2014: Cohn et al, 2007; Dowdy et al, 1998). Embedding a clinical ethicist in the interprofessional team is consistent with an evolving culture at our facility; from one where ethics consultation was a cumbersome process that required attendance of key leaders at formal meetings to one that provides real time consultation services where experts in ethics are available to clinicians on an as needed basis. In one landmark study, Schneiderman et al, 2003 demonstrated that inviting input from an ethics consultation service before conflict arose resulted in patients having significantly shorter lengths of stay in the ICU and fewer life-sustaining treatments for patients who ultimately did not survive their hospital stay, with nurses and physicians identifying the intervention as useful for addressing treatment conflicts.

More than 80 children die every year in our Pediatric Intensive Care Unit (PICU) and in more than $80 \%$ of those cases death follows an extended illness with either multiple hospitalizations and/or an extended period of time in the PICU. Medical and nursing leaders for our unit were concerned about perceived high levels of moral distress of staff. Our experience and a review of the literature suggested that delayed initiation of open honest discussion about limiting aggressive life sustaining interventions might be contributing to feelings that procedures and treatments were contributing to patient suffering and leading to moral distress in staff. This was particularly true when caring for patients with extended length of stay and who were at risk of death or significantly impaired functional recovery. To address this concern, we designed a practice innovation project. The aim of this project was to pilot a formal clinical ethics support program for the PICU team. A central element of this proposal was the exploration of how a proactive ethics approach supports healthcare providers and increases the likelihood that there 
would be open discussion about setting realistic care goals. This article reports the evaluation of this practice innovation project aimed at reducing moral distress of healthcare providers in a PICU. We evaluated the impact of the project on staff and on the target patient population (patients with extended ICU length of stay).

\section{Strategy:}

Setting and Sample: The hospital is considered a quaternary children's hospital and the largest provider for pediatric patients with complex medical conditions in the state. The PICU has 26 beds and during the 12 month data collection period had 1561 patient admissions. The hospital has a robust physician training program including a pediatric residency as well as a fellowship in pediatric critical care. There are more than 80 nurses employed full or part time on staff. It is worth noting that the medical and nursing leadership for the PICU had for several years supported staff participation in the organization's Unit Based Ethics Conversation program (Helft et al, 2009) ; establishing a strong trusting relationship between unit staff and the ethicist

Innovation: The innovation was a formal clinical mentoring and clinical ethics support program for clinicians in the form of inter-professional rounds. The pediatric critical care service elected to focus on patients with extended length of stay in part because multiple changes in attending physicians increases the chances of inconsistencies in management strategies for patients and delayed difficult discussions. Whether or not a patient was included in the discussion at PEACE rounds was based on clinical factors and parents were not included in the discussion as the focus was in part to coach clinicians on techniques for introducing sensitive topics related to the plan of care. Our goal was to address moral distress of staff and improve the quality of patient care.

\section{The intervention:}

Pediatric Ethics And Communication Excellence (PEACE Rounds) was designed to be a formal facilitated discussion about setting realistic care goals and the ethical issues inherent in caring for children with life-threatening illness. PEACE Rounds were process oriented and designed to clarify goals of treatment (Rehder et al, 2012) and whenever possible, achieve consensus. Over a twelve month period, once a week the interprofessional team met for PEACE Rounds. At each PEACE Rounds discussion the team completed a record of the discussion which was a modified version of a Decision Making Tool developed by Seattle Children's Hospital for each patient. (supplement A). 
During PEACE rounds, the Intensivist on service provided a very short synopsis of the medical plan for each child on the list. The senior intensivist helped focus the discussion on broad goals of treatment. The ethicist used probing questions to uncover situational risk factors for and early indicators of ethical conflict (Pavlish et al, 2011). The ethicist intentionally called on quiet members of the team, particularly non-physicians to invite them to share their perspective, particularly when values based discord was evident during discussions. Additionally, the ethicist provided just in time education and coaching using mnemonics such as NURSE (responding to emotion), ADAPT (discussing prognosis) and REMAP (transitions in goals of care) to illustrate effective communication techniques to use when engaging decision makers in sensitive discussions (Back et. al, 2009; Vital Talk, 2016).

Team members were prompted to discuss potential difficult medical and/or ethical aspects of management and when appropriate, explore rationale for considerations of limiting treatment interventions. The team considered if there was consensus that the child would survive the ICU stay, survive to hospital discharge and then would make specific recommendations about code status and overall goals of treatment. When there was consensus that a child's code status needed to be addressed with the family, a care conference was scheduled for the same week,

Dedicated discussion regarding realistic goals of care was a major focus. The bedside nurse and social worker provided insight regarding family expectations and understanding of the specific goals of care. All other disciplines involved in the care of the patient were also invited to attend (consulting medical services e.g cardiology or oncology, child life, chaplain services, respiratory therapy). When there was a perception of incongruence between predicted medical outcome and family expectations, a care conference was scheduled in the same week between the bedside medical team and family members. The leadership for the unit established the expectation that anyone involved in the care of a patient who was discussed at PEACE rounds should make attendance at PEACE rounds a priority, and if unable to attend at least speak with the attending physician to provide input for the discussion. Participation in the evaluation of the impact of the intervention on staff was voluntary.

Instruments: MoD was measured using two different instruments. The Moral Distress Scale Revised (MDS-R) is a valid and reliable tool to measure the presence of moral distress (Hamric, et al, 2012). It is a 21 item tool that asks respondents to rate their level of moral distress 
(frequency and intensity) related to specific situations. Participants were asked to complete the MDS-R pre implementation of the project and at the end of data collection (12 months later). The MDS-R was used as a measure of chronic MoD.

During the data collection period, healthcare providers were also asked intermittently to rate their MoD using the Moral Distress Thermometer (MDT). The MDT is a validated single item scale that simply defines moral distress then asks the respondent to rate on the scale their level of distress. (Wocial and Weaver, 2013) MoD was measured every other month using the MDT. For the purposes of this project the investigators chose to use an expanded version of the tool that gave respondents an opportunity to identify the factors that contributed to their sense of moral distress (supplement B). The MDT was used as a measure of acute MoD.

The PEACE Discussion Evaluation Form was created as a means to evaluate PEACE rounds participants' impression of the practice innovation. It is essentially a five point Likert scale (from 1 agree to 5 disagree) satisfaction survey with attention to the goals of the intervention (supplement C).

Procedures: This project was reviewed by our institution's IRB. Because patient inclusion was based on a standard practice innovation and historical matched controls, the IRB did not require consent from patients or their parents. At the request of the IRB, an informed consent statement was included in a written message any time healthcare providers were asked to rate their level of moral distress. Following IRB approval, one month prior to initiating the practice innovation healthcare providers practicing in the PICU were informed of the project via usual communication channels (e.g. e-mail, staff meetings). Information included explanation of the voluntary nature of participation in the evaluation of the impact of the project on provider moral distress. Healthcare providers were invited to complete the MDS-R electronically. Completion of the instrument satisfied our IRB requirement for informed consent to participate. All healthcare providers in the PICU were invited to complete the MDT intermittently during the data collection period. During the first and seventh months of data collection, participants were invited to complete an evaluation form immediately following attendance at PEACE rounds. Healthcare provider participants: Any clinician (physician, nurse, social worker, respiratory therapist, chaplain, pharmacist, child life, or music therapist) involved in the care of patients in the PICU was eligible to participate in the project. Due to the small number of non-physician or nurse participants, other providers were grouped in an "other" category (Table 1). There were 79 
nurses, 17 physicians and 35 other healthcare professionals who filled out the preimplementation questionnaires, 53 nurses, 13 physicians and 23 other healthcare professionals who filled out the post-implementation questionnaires, and 32 nurses and 10 physicians who filled out both pre and post-implementation questionnaires such that we were able to match their pre/post results.

Patient participants: Patients were identified for PEACE rounds discussions if the primary service responsible for their treatment plan was the critical care service and if they met any of the following criteria: 1) they had been in the PICU for more than 10 days consecutively; 2) they had been in the PICU for more than 10 days during the same hospital stay; or 3) any member of the team asked that the patient be discussed at PEACE rounds due to concerns about decreased therapeutic benefit of treatments. Patients were excluded from data collection if the primary medical service responsible for the plan of care was not the pediatric critical care service (e.g. surgical, cardiac, trauma, transplant patients were excluded). Once identified as eligible, patients were assigned a unique numeric identifier. Patient characteristics were selected for the purpose of determining the impact of the project on quality of patient care. The project team tracked the age, reason for admission, gender, ethinicity, PICU length of stay, code status, vent days, and mortality at hospital discharge, 30 days, and 365 days. Patient records were reviewed for the preceding 12 months to identify a historical comparison group for the patients discussed in PEACE rounds.

Patient demographic characteristics were recorded (Table 2). Reason for admission was categorized into respiratory, sepsis, cardiac, and other. The Pediatric Risk of Mortality (PRISM3) Score and Risk of Mortality were determined to assess the level of critical illness at PICU admission (Polluck, 1996). The PRISM-3 based predicted length of PICU stay was used to calculate the Indexed PICU LOS. Mortality at hospital discharge, 30 days, and 365 days were assessed.

Data Analysis: The distributions of moral distress assessed via MDT were recorded at five time points during a 12 month period and plotted on a graph over time for physicians and nurses. Repeated measures regression models were fit to compare clinician moral distress using the MDS-R. Models were fit for the MDS-R overall score and each of the individual items separately. Mean, standard deviation, and p-value of the statistical comparison of the pre and post MDS-R scores were calculated. 
Patient characteristics and medical outcomes between the project group and historical control group were compared. A minimum of 50 pairs of intervention and historical controls was necessary to provide $80 \%$ power to detect a 1 to 1.5 day difference in PICU stay with a standard deviation of 2.5 days. All analyses were completed using SAS software (9.4 (SAS Institute, Inc.) Chi-square test was used with categorical data while Wilcoxon rank-sum test was used for continuous measures. Significant difference was set at $p<0.05$. Proportions or median values with 25 th $/ 75$ th percentiles are reported.

Descriptive statistics were calculated for the overall satisfaction of the intervention as measured by results from the PEACE Discussion Evaluation Forms.

\section{RESULTS}

\section{Healthcare Provider Moral Distress:}

The MDS-R was used to measure MoD before the introduction of PEACE rounds and a 12 months after introduction. The numbers for "other" were small and represented a significantly heterogeneous group and therefor results will only be discussed for nurses and physicians. Overall, the scores are lower (indicating a drop in moral distress) for respondents on all items, however with few exceptions, not in a statistically significant way (Table 3). In the matched comparison (same person's pre/post score) the item "Feel pressure to order what I consider to be unnecessary tests and treatments" showed significantly improved moral distress for physicians. For the aggregate comparison, no items were significantly improved for physicians.

There were three items on the instrument that showed statistically significant improvement in moral distress for nurses for both matched and aggregate data comparisons. On the aggregated comparison for nurses, four additional items showed a statistically significant drop in moral distress. When compared, there were no significant differences between physicians' and nurses' mean moral distress scores pre or post intervention as measured by the MDS-R.

The MDT was used to measure "acute" moral distress. The level of moral distress was measured every two months. The mean score fluctuated, showing month to month variability (Figure 1). "Clinical Situations" represented the single most frequent contributing factor to moral distress (Table 4). For each time point measured, the median MDT score is below the mean, suggesting that there are outliers with higher moral distress scores skewing the mean. 
However, over the course of the intervention, the range of scores for physicians and nurses narrowed, with a lower maximum score suggesting that there were fewer individuals with higher levels of moral distress (fewer outliers).

\section{Patient population:}

Patient outcomes showing statistically significant differences between patients discussed on PEACE rounds and their historical controls included a decrease in PRISM indexed PICU length of stay (4.94 control vs 3.37 PEACE, $\mathrm{p}=0.015)$ ), and an increase in code status changes to DNR ( $11 \%$ control vs $28 \%$ PEACE, $p=0.013)$. There were no differences in 30 or 365 -day mortality despite an increase in in-hospital death ( $9 \%$ control vs $25 \%$ PEACE, $\mathrm{p}=0.015$ ). (Table 5). There were no statistically significant differences in clinical characteristics in the subgroup of patients who died in the control and intervention groups.

\section{Evaluation of PEACE Rounds}

When evaluating PEACE rounds, the response was overwhelming with regards to improvement in communication. When asked if the PEACE rounds helped participants improve their ability to communicate with the patient/family, $85.5 \%$ of nurses, $95.1 \%$ of physicians and $96.2 \%$ of other clinical care providers indicated yes. When asked if the PEACE rounds helped participants improve their ability to communicate with other members of the health care team, $88.2 \%$ of nurses, $90.2 \%$ of physicians and $98.3 \%$ of other clinical care providers indicated yes.

\section{Discussion:}

Acute levels of moral distress as measured by the MDT fluctuated from month to month, with "clinical situations" representing the single most common contributing factor. This suggests that moral distress is directly and significantly impacted by the inpatient population at any given point in time. Interestingly, mean distress scores were consistently higher than median scores, suggesting the presence of high distress outliers skewing the mean. This finding, in context with lower maximum distress scores during the intervention, suggests fewer high distress outliers during the intervention period. Considering that high moral distress is associated with leaving a position (Allen et al, 2013; Papathanassoglou, et al, 2012; Piers, et al, 2011, Trotochaud et al, 2015;), identifying outliers who have high levels of moral distress might be more important than identifying a drop in mean scores of a group of healthcare providers. Our results indicate that tracking moral distress in real time may provide an opportunity to identify outliers who could benefit from an intervention. 
Moral distress as measured by the MDS-R was more difficult to interpret. While we were able to match some individuals' pre and post measurements, we were not able to track and identify how frequently (if at all) these individuals attended PEACE Rounds. We can state that the PEACE Rounds seemed to have a greater impact on nurses' moral distress when compared to physicians. One of the three items that showed a statistically significant drop in moral distress for nurses for both matched and aggregate comparisons "Witness diminished patient care quality due to poor team communication." is striking in that the most frequently identified contributing factor to nurses' moral distress on the MDT was "Inadequate communication among team members.".

Unlike a recent study by Andereck et al, we were able to demonstrate a decrease in ICU length of stay for patients (2016). This is noteworthy as it is the first reported study to demonstrate this outcome for a pediatric population. At first glance, it may appear that PEACE rounds resulted in higher mortality. However, a closer examination of the long-term outcome between the historical controls and the intervention group at 30 and 365 days suggests that PEACE rounds led to more proactive conversation with families attempting to set realistic goals of care. This is evidenced by a change in code status that occurred twice as frequently in the intervention group ( $28 \%$ versus $11 \%, \mathrm{p} 0.01)$. Our results suggest that PEACE rounds may have helped the medical team achieve consensus on therapeutic benefit of treatments and engage with patients' families sooner in conversations about realistic goals of care as well as the process of making difficult decisions.

Concerning perceived intervention quality, respondents were uniform in their belief that PEACE Rounds improved communication. This is notable as the respondents were expected to attend PEACE Rounds if they were an assigned care provider for a patient who was being discussed and the response rate for these surveys was greater than $90 \%$. As in previous work (Bruce et al, 2014; Cohn et al, 2007), clinicians appreciated the proactive ethics approach; Greater than $85 \%$ of nurses (85.5), physicians (95.1), and other team members (96.2) indicated improved ability to communicate with patient/family. Similarly, respondents $(88.2 \%$ of nurses, $90.2 \%$ of physicians, and $98.3 \%$ of other team members) identified improved communication within the medical team. The structure and goals of PEACE Rounds are consistent with what has been identified as an intervention for managing moral distress, namely group discussion and ethical rounding (Wilson et al, 2013). 
We were disappointed that there was not a statistically significant decrease in moral distress scores. We suspect the major contributing factor for this is that some of the most distressing patient cases involved patients who were not discussed at PEACE rounds. In the future, it would be important to tie MDT scores to specific patient case discussions to more clearly determine if PEACE rounds have the desired impact on provider moral distress. Despite the lack of statistical evidence, the success of PEACE rounds is evident in that they continue in our PICU, with inclusion of patients from additional service lines and have been expanded to the neonatal intensive care unit. The pediatric palliative care team attends rounds weekly. Ethics no longer attends weekly, however attends at the request of the team when there are concerns for ethical conflict.

\section{Evaluation Limitations:}

The evaluation of our practice innovation was challenged with several limitations. Assessing moral distress every two months may be less frequent than ideal for assessing trends, as this approach yields only 6 data points per year. Additionally, clinical situations vary dramatically from day to day so evaluating moral distress intermittently over a 12 month period undoubtedly means the clinical triggers for moral distress were not necessarily comparable. Compounding this complexity was the fact that healthcare providers whose moral distress was being measured were caring for patients who were co-managed and not part of the intervention cohort. Most notably, surgical patients are co-managed with the critical care service, a situation where divergent clinical management points of view are likely to occur. With regards to moral distress of team personnel, a concession must be made that some clinical situations will be distressing regardless of team dynamics. As a result, (and supported by the "clinical situations" variable) changes in distress scores may better reflect patient exposure than effect of the PEACE intervention.

With regards to patient outcomes, though each collection period for PEACE rounds (60) and historical control (66) patients encompassed twelve months, the different size of the two groups certainly affects power for the data analysis. This subset of patients in the ICU setting at our facility encompasses a mere $3 \%$ of the total population cared for on a yearly basis. Second, and perhaps more important, this study did not follow patients longitudinally, as would be the case in a cohort evaluation of this practice innovation. It is worth considering whether the greatest impact of this type of intervention lies in its effect on the approach to long term care for 
these children, as establishing set care plans for a patient prior to departure from the PICU may ultimately improve family understanding of the child's needs and limitations, quality of life, and more appropriate resource utilization as an outpatient and during future hospital admissions.

Despite this project's multiple limitation, the results indicate an opportunity for future research and evaluation of the innovation. It might be more useful to measure levels of moral distress immediately pre and post PEACE rounds discussions and ask respondents to identify their level of moral distress related to specific patients' plan of treatment.

\section{Conclusion:}

Dedicated medical team conferences to establish unified goals of care and discuss challenging ethical issues for patients with prolonged PICU stays can positively impact healthcare provider moral distress through improved communication and can improve overall patient care. Some factors that contribute to moral distress are related to patients in a unit suggesting that moral distress should be monitored on a regular basis. Improved patient care was manifested as decreased PRISM indexed PICU LOS and an increase in code status changes in favor of treatment limitation, without a corresponding increase in 30 and 365 day mortality. We believe patient care is improved when establishing goals of treatment for long-stay PICU patients includes intentional discussion of clinically and ethically challenging issues. 


\section{References}

Allen, R., Judkins-Cohn, T., deVelasco, R., Forges, E., Lee, R., Clark, L., et al. (2013). Moral distress among healthcare professions at a health system. JONA'S Healthcare Law, Ethics and Regulation, 15, 111-118.

American Academy of Pediatrics, Committee on Bioethics (1996). Ethics and the care of critically ill infants and children. Pediatrics, 98(1), 149-152.

Andereck, W. S., McGaughey, W., Schneiderman, L. J. \& Jonsen, A. R. (2016). Seeking to reduce non-beneficial treatment in the ICU: An exploratory trial of proactive ethics intervention. Critical Care Medicine, 42(4), 824-830.

Back AL, Arnold RM, Baile WF, Tulsky JA, Barley GE, Pea RD, Fryer-Edwards KA. (2009). Faculty development to change the paradigm of communication skills teaching in oncology. $J$ Clin Oncol. 27(7):1137-41. doi: 10.1200/JCO.2008.20.2408.

Bruce, C. R., Miller, S. M., \& Zimmerman, J. L. (2015). A qualitative study exploring moral distress in the ICU team: the importance of unit functionality and intrateam dynamics. Crit Care Med. 43(4), 823-31. doi: 10.1097/CCM.0000000000000822.

Bruce, C. R., Peñaa, A., Kusina, B. B., Allena, N. G., Smith, M. L., \& Majumdera, M. A. (2014). An Embedded Model for Ethics Consultation: Characteristics, Outcomes, and Challenges. AJOB Empirical Bioethics. 5(3), 8-18. DOI:10.1080/23294515.2014.889775

Clark, J. D. \& Dudzinski, D. M. (2013). The Culture of Dysthanasia: Attempting CPR in Terminally Ill Children. Pediatrics 131(3), 572-580. (doi: 10.1542/peds.2012-0393)

Cohn, F., Goodman-Crews, P., Todman, W., Schneiderman, L. \& Waldman, E. (2007). Proactive ethics consultation in the ICU: A comparison of value perceived by healthcare professionals and recipients. The Journal of Clinical Ethics, 18(2), 140-147.

Dowdy, M. D., Robertson, C, \& Bander, J. A. (1998). A study of proactive ethics consultation for critically and terminally ill patients with extended lengths of stay. Critical Care Medicine 26(2), 252-259.

Elpern, E. H., Covert, B. \& Kleinpel, 1. R. (2005). Moral distress of staff nurses in a medical intensive care unit. American Journal of Critical Care 14(6), 523-530.

Fourie, C. (2015). Moral distress and moral conflict in clinical ethics. Bioethics. 29(2), 91-7. doi: 10.1111/bioe.12064. Epub 2013 Nov 8.

Garros, D., Austin, W., \& Carnevale, F. A. (2015). Moral Distress in Pediatric Intensive Care. JAMA Pediatr: 169(10):885-6. doi: 10.1001/jamapediatrics.2015.1663.

Halpern, S. D., Becker, D., Curtis, J. R., Fowler, R., Hyzy, R., Kaplan, L. J., Rawat, N., Sessler, C. N., Wunsch, H., \& Kahn, J. M. (2014). An Official American Thoracic Society/American Association of Critical-Care Nurses/American College of Chest Physicians/Society of Critical 
Care Medicine Policy Statement: The Choosing Wisely® Top 5 List in Critical Care Medicine. American Journal of Respiratory and Critical Care Medicine, 190( 7), 818-826. doi: 10.1164/rccm.201407-1317ST.

Halpern, S. (2011). Perceived Inappropriateness of Care in the ICU. What to Make of the Clinician's Perspective? JAMA, 306(24), 2725-2726. doi:10.1001/jama.2011.1897.

Hamric, A. B., \& Blackhall, L. J. (2007). Nurse-physician perspectives on the care of dying patients in intensive care units: Collaboration, moral distress and ethical climate. Critical Care Medicine 35(2), 422-429.

Hamric A. B., Borchers, C. T., \& Epstein, E. G. (2012). Development and testing of an instrument to measure moral distress in health care professionals. AJOB Primary Research, 3(2), $1-9$.

Helft, P.R., Bledsoe, P.D., Hancock, M., Wocial, L.D. (2009). Facilitated ethics conversations: a novel program for managing moral distress in bedside nursing staff. JONAS Healthc Law Ethics Regul. 11(1), 27-33.

Houston, S., Casanova, M. A., Leveille, M., Schmidt, K. L., Barnes, S. A., Trungale, K. R., \& Fine, R. L. (2013). The intensity and frequency of moral distress among different healthcare disciplines. J Clin Ethics. 24(2), 98-112.

Lamiani, G, Borghi, L \& Argentero, P. (2015). When healthcare professionals cannot do the right thing: A systematic review ofmoral distress and its correlates. Journal of Health Psychology, 1-17. doi: 10.1177/1359105315595120

Kalvemark, S., Hoglund, A., Hansson, M., Westerholm, P. \& Arnetz, B. (2004). Living with conflicts-ethical dilemmas and moral distress in the health care system. Social Science \& Medicine 58(6), 1075-1084.

Karanikola, M. N., Alarran, J. W., Drigo, E., Giannakopoulou, M., Kalafati, M., Mpouzika, M., Tsiaousis. G, Z., \& Papathanassogiou, E. D. (2014). Moral distress, autonomy and nursephysician collaboration among intensive care unit nurses in Italy. Journal of Nursing Management. 22, 472-484. DOI: 10.1111/jonm.12046

McAndrew, N. S., Leske, J. S., \& Garcia, A. (2011). Influence of Moral Distress on the Professional Practice Environment During Prognostic Conflict in Critical Care. Journal of Trauma Nursing: 18(4): pp. 221-230. doi: 10.1097/JTN.0b013e31823a4a12

Mekechuk, J. (2006). Moral distress in the pediatric intensive care unit: the impact on pediatric nurses. Int J Health Care Qual Assur Inc Leadersh Health Serv. 19(4-5), i-vi.

Mobley, M. J/, Rady, M. Y., Verheijde, J. L., Patel, B., \& Larson, J. S. (2007). The relationship between moral distress and perception of futile care in the critical care unit. Intensive Crit Care Nurs 23(5), 256-63. Epub 2007 Aug 6. 
Musto, L. C., Rodney, P. A., \& Vanderheide, R. (2015). Toward interventions to address moral distress: Navigating structure and agency. Nursing Ethics 22(1), 91-102. Doi:

10.1177/0969733014534879.

Pavlish, C, Brown-Saltzman, K, Hersh, M, Shirk, M, and Nudelman, O. (2011). Early indicators and risk factors for ethical issues in clinical practice. Journal of Nursing Scholarship, 43(1), 13-21.

Papathanassoglou, E. D., Karanikola, M. N., Kalafati, M., Giannakopoulou, M., Lemonidou, C, \& Albarran, J. W. (2012). Professional autonomy, collaboration with physicians, and moral distress among European intensive care nurses. Am J Crit Care. 21(2):e41-52. doi: 10.4037/ajcc2012205.

Piers, R. D. Azoulay, E., Ricou, B., Dekeyser Ganz, F., Decruyenaere, J., Max ,A., Michalsen, A., Maia, P. A., Owczuk, R, Rubulotta, F., Depuydt, P., Meert, A. P., Reyners, A. K., Aquilina, A., Bekaert, M., Van den Noortgate, N. J., Schrauwen, W. J., \& Benoit. D, D. (2011).

Perceptions of appropriateness of care among European and Israeli intensive care unit nurses and physicians. JAMA 306(24), 2694-2703.

Polluck, M. M., Patel, K. M., \& Ruttimann, U. E. (1996). PRISM III: An updated pediatric risk of mortality score. Critical Care Medicine, 24(5), 743-752.

Rehder, K. J., Uhl, T. L., Meliones, J. N., Turner, D. A., Smith, P. B. \& Mistry, K. P. (2012). Targeted interventions improve shared agreement of daily goals in the pediatric intensive care unit. Pediatr Crit Care Med 13(1), 6-10.

Schneiderman, L. J., Gilmer, T., Teetzel, H. D., Dugan, D. O., Blustein, J., Cranford, R., et al. (2003). Effect of ethics consultations on nonbeneficial life-sustaining treatments in the intensive care setting: A randomized controlled trial. JAMA 290, 1166-1172

SAS 9.4 (2014). SAS Institute Inc., Cary, NC, USA.

Trotochaud, K, Coleman, J. R., Krawiecki, N., \& McCracken, C. (2015). Moral Distress in Pediatric Healthcare Providers. J Pediatr Nurs. 2015 Apr 10. pii: S0882-5963(15)00082-2. doi: 10.1016/j.pedn.2015.03.001. [Epub ahead of print]

Ulrich, C. M., Hamric, A. B. \& Grady, C. (2010). Moral distress: A growing problem in the health professions. Hastings Center Report 40(1), 20-22.

Vital Talk, 2016. http://vitaltalk.org/quick-guides.

Wall, S., Austin, W. J., \& Garros, D. (2015). Organizational influences on health professionals' experiences of moral distress in PICUs. HEC Forum DOI 10.1007/s10730-125-9166-8.

Whitehead, P. B., Herbertson, R. K., Hamric, A. B., Epstein, E. G., and Fisher, J. M. ( 2015). Moral distress among healthcare professionals: report of an institution-wide survey. Journal of Nursing Scholarship 47(2), 117-25. doi: 10.1111/jnu.12115. Epub 2014 Nov 29. 
Wilson, M. A, Goettemoeller, D. M., Bevan, N. A., \& McCord, J. M. (2013). Moral distress: levels, coping and preferred interventions in critical care and transitional care nurses. J Clin Nurs. 22(9-10):1455-66. doi: 10.1111/jocn.12128. Epub 2013 Mar 8.

Wocial, L. D. \& Weaver, M. T. (2013). Evaluation of a new tool for Measuring Moral Distress: The Moral Distress Thermometer. J Adv Nurs. 69(1), 167-74. doi: 10.1111/j.13652648.2012.06036.x.

Wocial, L. D., Hancock, M., Bledsoe, P.D., Chamness, A., \& Helft, P.R. (2010). An Evaluation of Unit Based Ethics Conversations. JONA's Healthcare Law, Ethics and Regulation 12(10), 48-54. 